\title{
Colonic Gallstone Ileus: Treatment Challenges
}

Review began 09/30/2021 Review ended 11/20/2021 Published 11/24/2021

๑) Copyright 2021

Da Cunha et al. This is an open access article distributed under the terms of the Creative Commons Attribution License CCBY 4.0., which permits unrestricted use, distribution, and reproduction in any medium, provided the original author and source are credited.

\author{
Teresa Da Cunha ${ }^{1}$, Bashar Sharma ${ }^{2}$, Steven Goldenberg ${ }^{2}$ \\ 1. Internal Medicine, University of Connecticut Health, Farmington, USA 2. Gastroenterology and Hepatology, \\ University of Connecticut Health, Farmington, USA
}

Corresponding author: Teresa Da Cunha, dacunha@uchc.edu

\begin{abstract}
Intestinal obstruction at the level of the colon is rarely caused by a gallstone. Colonic gallstone is more frequently observed in elderly patients and is associated with high mortality due to treatment challenges. Management with less invasive approaches, including mechanical lithotripsy and endoscopy has been evolving. However, the outcomes are variable, and surgery remains the main cornerstone of treatment. We present a case of an 89-year-old male with gallstone ileus at the level of the sigmoid colon in whom treatment with endoscopy was not successful. We performed an extensive review of the literature to understand the most common presentation, diagnostic modalities, and treatment approach of the sporadic reported cases of colonic gallstone ileus.
\end{abstract}

Categories: Gastroenterology, General Surgery

Keywords: cholecystoenteric fistula, gallstone, bowel obstruction, colonic gallstone ileus, gallstone ileus

\section{Introduction}

Gallstone ileus is a well-characterized pathology in which bowel obstruction occurs secondary to a gallstone impaction. The stone usually travels to the intestine through a cholecystoenteric fistula which typically forms between the gallbladder and the duodenum, but it can form with other sites of the gastrointestinal tract. The most common site of stone impaction is the ileum, however, around $4 \%$ can occur in the colon [1]. Elderly patients and especially females are at higher risk for developing colonic gallstone ileus, moreover, they usually present with other comorbidities which contribute to the high morbidity and mortality associated with colonic gallstone ileus. The management of colonic gallstone ileus has been evolving to less invasive approaches with some success with therapeutic colonoscopy, however, surgery remains the cornerstone for its management [2]. We present a case of colonic gallstone ileus and performed an extensive review of the literature to understand the most common presentation, diagnostic modalities, and treatment approach of the sporadic reported cases of colonic gallstone ileus.

\section{Case Presentation}

An 89-year-old male with a history of stroke, atrial fibrillation, heart failure, esophageal adenocarcinoma status post radiation, cholecystitis one year prior to presentation (treated with percutaneous cholecystostomy tube that had subsequently been removed), presented to the emergency room with left lower quadrant abdominal pain of two-day duration. He denied nausea, vomiting, diarrhea, constipation, or blood in the stool. He did not have any sick contacts or recent travel. In the emergency room, his temperature was $98.1^{\circ} \mathrm{F}$, pulse 80 beats per minute, respiratory rate 16 breaths per minute, and blood pressure 106/67 mmHg. The laboratory blood tests are described in Table 1. 


\section{Cureus}

\begin{tabular}{|c|c|c|}
\hline Blood test & Result & Normal range \\
\hline WBC $\left(10^{9} / L\right)$ & 8.3 & $4.5-11$ \\
\hline Hemoglobin (g/dL) & 11.5 & 13.5-17.5 \\
\hline Hematocrit (\%) & 35 & $41-51$ \\
\hline Platelet count $\left(10^{3} / \mathrm{mL}\right)$ & 326 & $150-450$ \\
\hline Sodium (mEq/L) & 136 & $135-145$ \\
\hline Potassium (mEq/L) & 4.8 & $3.6-5.2$ \\
\hline Creatinine (mg/dL) & 1.0 & $0.74-1.35$ \\
\hline BUN (mg/dL) & 21 & $6-24$ \\
\hline AST (U/L) & 20 & $8-33$ \\
\hline ALT (U/L) & 9 & $7-55$ \\
\hline ALP (U/L) & 176 & $44-147$ \\
\hline TBIL (mg/dL) & 0.7 & $0-1.0$ \\
\hline
\end{tabular}

\section{TABLE 1: Blood test results on admission.}

WBC, white blood cell; BUN, blood urea nitrogen; AST, aspartate aminotransferase; ALT, alanine aminotransferase; ALP, alkaline phosphatase; TBIL, total bilirubin

A CT of the abdomen and pelvis revealed a fistula between the gallbladder and the large bowel at the hepatic flexure and air in the gallbladder. Marked diverticulosis with wall thickening and fat stranding was seen at the level of the mid to distal descending colon. A large lamellated stone $(3 \mathrm{~cm} \mathrm{x} 2.6 \mathrm{~cm})$ was present in the distal descending colon (Figure 1). A gastrografin enema revealed sigmoid colon diverticular stricturing disease (Figure 2).

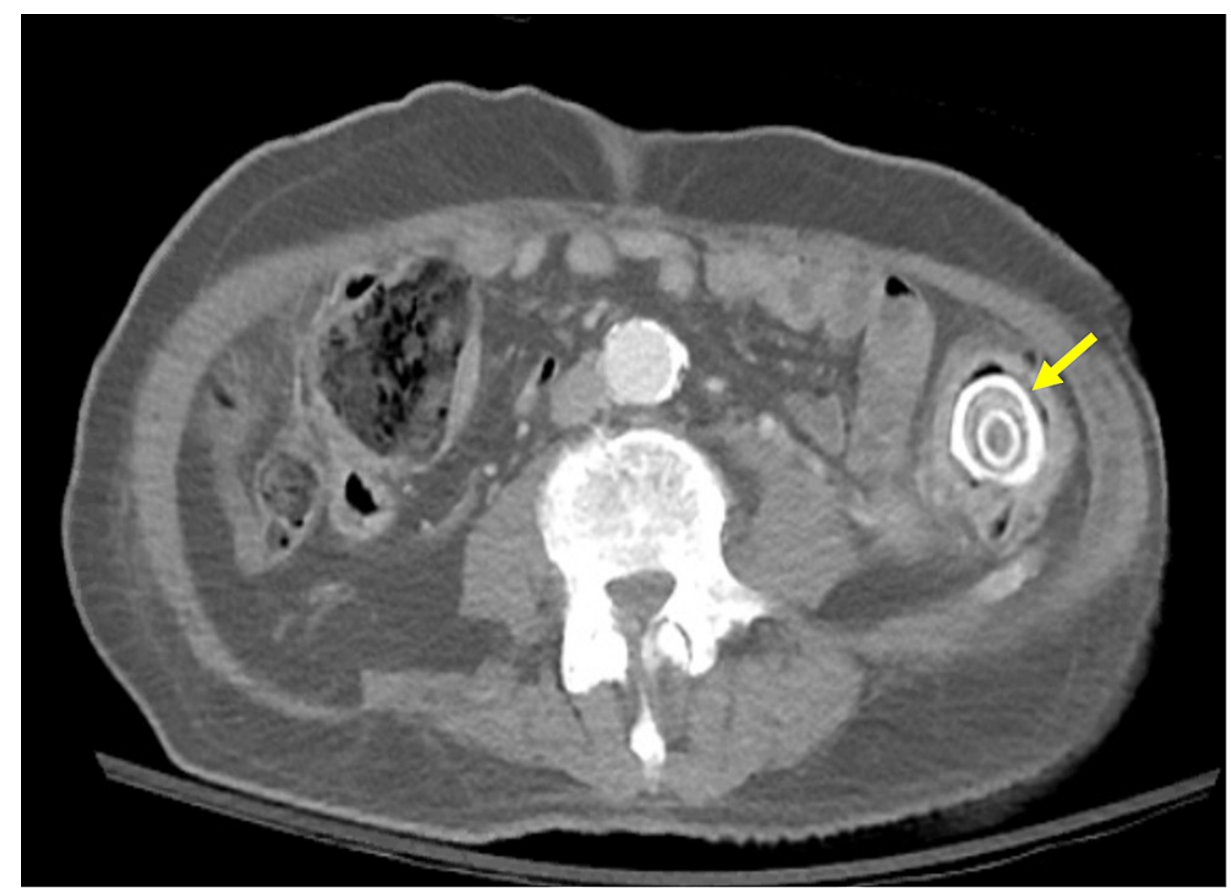

FIGURE 1: CT abdomen pelvis shows large lamellated stone in the distal descending colon. 


\section{Cureus}

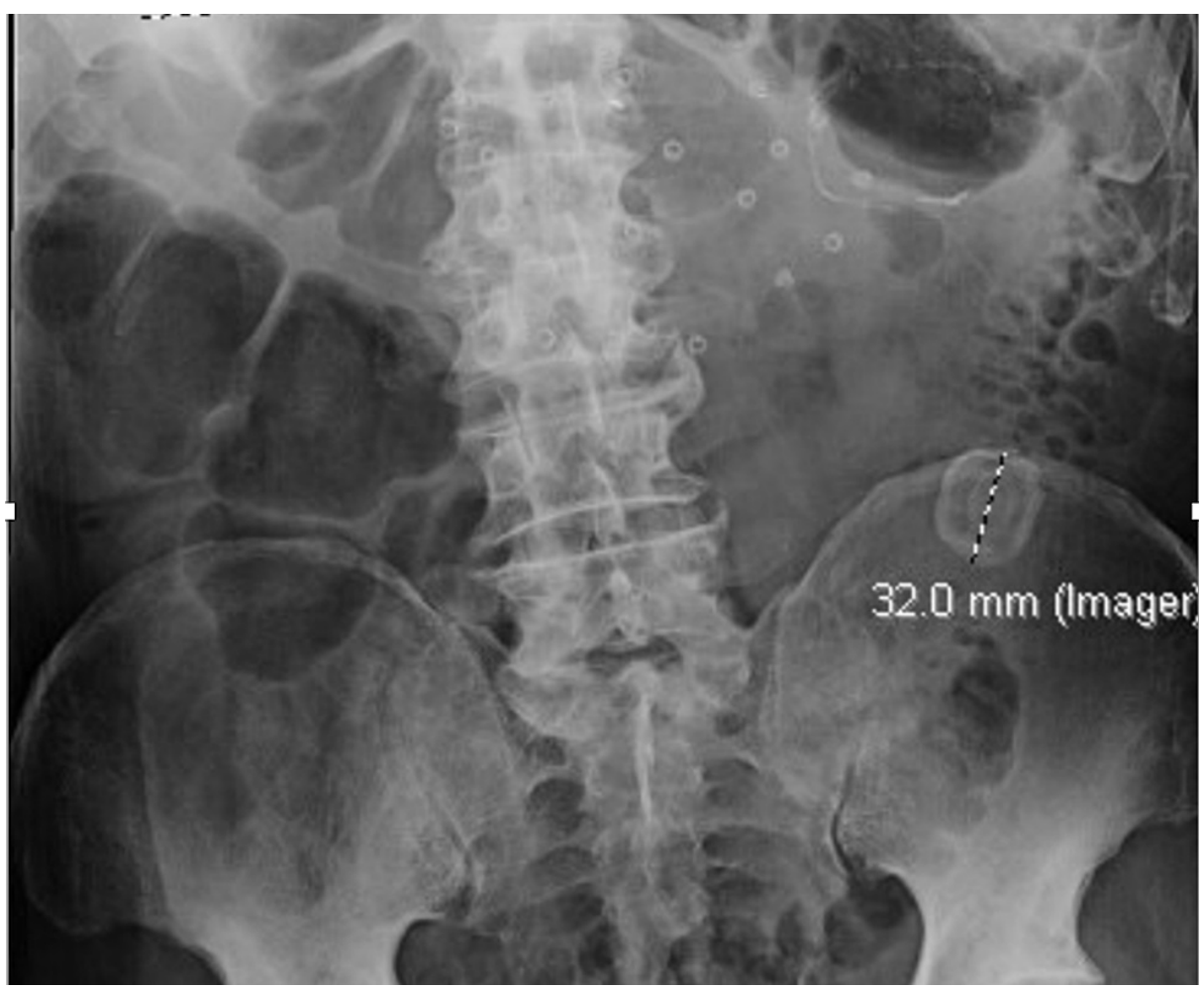

FIGURE 2: Gastrografin enema showing diverticular structuring and a $32 \mathrm{~mm}$ calculus in the descending colon.

Given the patient's several comorbidities surgery was deferred, the patient was kept nil per mouth and conservative treatment with an aggressive bowel regimen including mineral oil enemas as well as IV hydration was started. However, after four days he did not have any bowel movement and for this reason, total parenteral nutrition was initiated and an endoscopic approach to remove the stone was planned. On the fifth day of hospitalization, a colonoscopy was performed which showed a large black pigmented stone that was completely obstructing the lumen of the sigmoid colon, the surrounding mucosa appeared ulcerated (Figure 3).

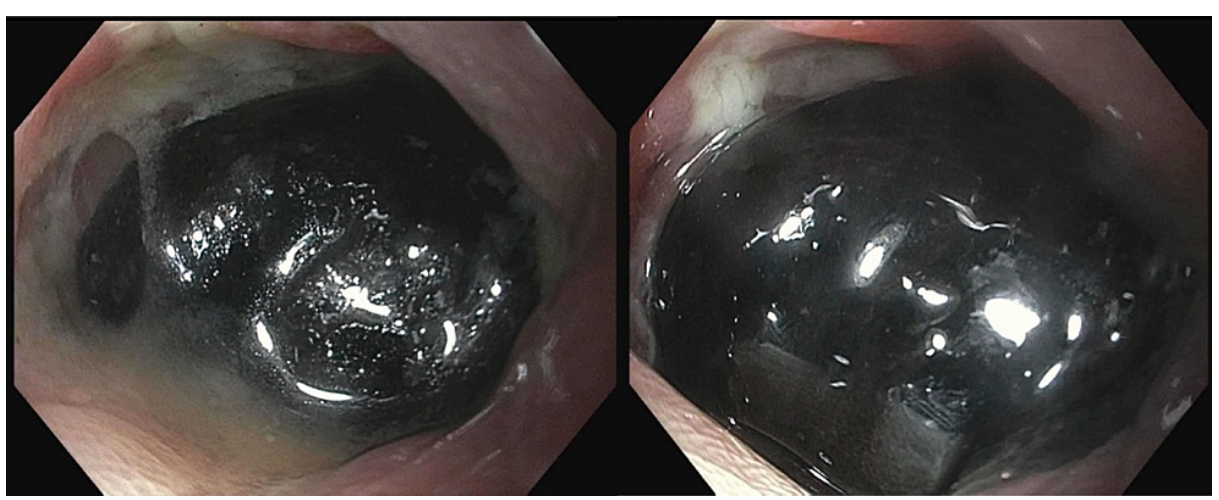

FIGURE 3: Large pigmented gallstone seen at the sigmoid level during colonoscopy.

Attempts to pass a guidewire proximal to the stone to pursue balloon-assisted dislodgment of the stone or mechanical lithotripsy as well as attempts to capture the stone in a retrieval net were unsuccessful. Consequently, an exploratory laparotomy with partial left colectomy was successfully done, however, the cholecystocolonic fistula was left intact. Later, the pathology results revealed a $3.4 \mathrm{~cm} \times 2.7 \mathrm{~cm}$ black stone and a $4.5 \mathrm{~cm} \times 3 \mathrm{~cm}$ transmural defect at the colonic wall where the stone was impacted. The patient's postoperative period was complicated, he did not tolerate oral intake and his nutrition deteriorated substantially despite total parenteral nutrition (TPN). At the request of the patient and the family he opted for inpatient hospice care. Unfortunately, he died 15 days after the surgery. 


\section{Cureus}

\section{Discussion}

Gallstone ileus is an extremely rare cause of mechanical bowel obstruction and is responsible for less than $5 \%$ of cases of bowel obstruction. However, when stratifying according to age, it is the main cause of intestinal obstruction in up to $25 \%$ of patients older than 65 [1]. Moreover, about $0.3 \%-0.5 \%$ of patients with cholelithiasis develop gallstone ileus [3]. Both the study of Rodríguez Hermosa et al. and Clavien et al. reported a higher incidence of this pathology in elderly women with male to female ratios of 5.5:1 and 8.25:1 and a mean age of 76 and 78 years old, respectively [4-5]. These studies, however, lack power owing to its sample size. A recent large study at a national level in the United States showed a lower incidence $(0.095 \%)$ of gallstone ileus in 3.452 .536 cases of mechanical bowel obstruction, from these, more than $70 \%$ were elderly women [6]. In the study of Rodríguez Hermosa et al. which reviewed 40 cases of gallstone ileus, around $65 \%$ of patients had a history of biliary disease, including cholecystitis, biliary colic, cholelithiasis, or choledocholithiasis. In most cases, a biliary-enteric fistula develops allowing communication between the gallbladder and the intestine and subsequent travel of the gallstones [4]. The most frequent type is a cholecystoduodenal fistula, due to the anatomical proximity [3]. Nonetheless, a cholecystocolonic fistula has been reported and it is the most common association with gallstone impaction in the colon [7].

The location of the gallstone entrapment can occur anywhere from the duodenum to the colon. According to the study of Rodríguez Hermosa et al. the most common location of the gallstone in the gastrointestinal tract was the ileum (62.5\%), followed by the jejunum (22.5\%), duodenum (7.5\%), and colon (2.5\%) [4]. However, in the larger study of Reisner et al. that involved 1001 cases of ileus, the ileum also accounted for $60.5 \%$ of the cases but a higher percentage of patients (4.1\%) had had a colonic gallstone impaction leading to bowel obstruction [1]. The symptomatology of gallstone ileus varies according to the location of the obstruction. Symptoms of intestinal obstruction are commonly reported, others include jaundice and hematemesis. In the case of colonic gallstone ileus, constipation, abdominal pain, and vomiting are frequently reported [7-9].

We performed a review of the literature to further understand the clinical presentation, diagnosis, treatment, and outcomes of patients with gallstone ileus of the colon. EMBASE and PubMed were used to search for articles related to bowel obstruction caused by gallstone impaction in the colon. The following keywords were used: "colonic gallstone ileus," "gallstone impaction,” "gallstone bowel obstruction,” "sigmoid gallstone ileus," and "cholecystocolonic fistula." The references of retrieved articles were extensively reviewed for further inclusion of additional cases. A total of 56 cases were found and we also included our case in this review.

The mean age was 80 years old and $74 \%(n=42)$ were women. The most commonly reported clinical presentation was abdominal pain (84\%), vomiting (67\%), and constipation (61\%). At least $25 \%$ of the patients had a reported history of some type of biliary tract disease including, cholecystitis, symptomatic cholelithiasis, and cholangitis. Elevated inflammatory markers are frequent, from the 22 cases that mentioned the initial blood results, the white blood count ranged from $6.7 \times 109 / \mathrm{L}$ to $27.4 \times 109 / \mathrm{L}$ and the average was $14.3 \times 109 / \mathrm{L}$. Similarly, from the 16 patients who had a reported C-reactive protein level, 15 had elevated levels which averaged $122 \mathrm{mg} / \mathrm{L}$.

Given the clinical picture of bowel obstruction, abdominal X-rays are usually performed and the Rigler's triad (small bowel obstruction, gallstone outside the gallbladder, air in the bile ducts) is sometimes present, however, a nonspecific dilation of the small and large bowel is frequent. For this reason, CT of the abdomen is performed most of the times and $81 \%(n=46)$ of cases were diagnosed by CT scan. In some cases, due to the hemodynamic instability, emergent laparotomy was required, for this reason in $7 \%(n=4)$ of cases the diagnosis was done during surgery. Other diagnostic modalities were plain abdominal X-ray, endoscopy and gastrografin study, each of these were the main diagnostic methods in only $5 \%(n=3), 5 \%(n=3)$, and $2 \%(n=1)$ of cases, respectively (Table 2).

\begin{tabular}{|l|c|}
\hline Main diagnostic modality & N (\%) \\
\hline CT abdomen & $46(81)$ \\
Laparotomy & $4(7)$ \\
Abdominal X-ray & $3(5)$ \\
Endoscopy & $3(5)$ \\
Gastrografin & $1(2)$ \\
\hline
\end{tabular}

TABLE 2: Main final diagnostic modalities.

The main final diagnostic modality reported by each individual study that provided the most accurate diagnosis. 


\section{Cureus}

The location of the gallstone impaction in the colon was the sigmoid colon, rectosigmoid, or at the descending colon/sigmoid junction in $86 \%(n=49)$ of cases, followed by the descending colon $(n=4)$ and transverse colon $(n=3)$ (Table 3). This could be explained by the higher frequency of diverticulosis at the sigmoid segment and consequent increased risk for strictures [10]. The presence of a biliary enteric fistula was described in 40 cases, of which 37 were cholecystocolonic fistulas and three were cholecystoduodenal. The majority of the cholecystocolonic fistulas were formed at the hepatic flexure, including ours. The stone size varied, when using the largest reported diameter, it ranged from 2 to $7 \mathrm{~cm}$, with an average of $4.24 \mathrm{~cm}$.

\begin{tabular}{|l|l|}
\hline Stone location & N (\%) \\
\hline Sigmoid / descending sigmoid junction / rectosigmoid & $49(86)$ \\
\hline Descending colon & $4(7)$ \\
\hline Transverse colon & $3(5)$ \\
\hline
\end{tabular}

TABLE 3: Location of the stone in the colon.

Similar to gallstone ileus occurring at the small intestine, the mainstay of treatment in colonic gallstone ileus is enterolithotomy which consists of a longitudinal colotomy proximal to the point of stone impaction followed by stone removal and closure of the colotomy, however, partial colectomy is sometimes needed and Hartmann's procedure is common. Owing to advances in endoscopic procedures, the use of colonoscopy for stone removal has been increasing, nevertheless with variable success. Of the 57 cases, 40 (70\%) were treated with laparotomy of which four died during the hospitalization, a total of 32 patients had an attempted colonoscopy for treatment but it was only successful in removing the stone in eight patients (14\%) and one patient died. Two patients had successful treatment with laparoscopy, one with trephine loop colostomy, five patients had conservative treatment, and one case did not clarify the type of surgery (Table 4). Table 5 summarizes the main characteristics of reported cases of colonic gallstone ileus.

\begin{tabular}{|l|l|}
\hline Treatment & N (\%) \\
\hline Laparotomy & $40(70)$ \\
Endoscopy & $8(12)$; attempted in $32(56)$ \\
Conservative & $5(9)$ \\
Laparoscopy & $2(4)$ \\
Trephine loop colostomy & $1(2)$ \\
\hline
\end{tabular}

TABLE 4: Final treatment modalities.

\begin{tabular}{|c|c|c|c|c|c|c|c|}
\hline Study & Age & Gender & Diagnosis & Location & $\begin{array}{l}\text { Stone size } \\
(\mathrm{cm})\end{array}$ & Final treatment & Outcome \\
\hline 1 & 82 & Male & CT abdomen & Sigmoid & 7 & Laparotomy & Discharged \\
\hline 2 & 90 & remale & $\begin{array}{l}\text { Gastrografin } \\
\text { enema }\end{array}$ & Sigmoid & 3.2 & Laparotomy & Discharged \\
\hline 3 & 87 & Female & CT abdomen & Sigmoid & 5 & Laparotomy & Discharged \\
\hline 4 & 72 & Female & CT abdomen & Sigmoid & 2.2 & Laparotomy & Discharged \\
\hline 5 & 85 & Female & CT abdomen & $\begin{array}{l}\text { Descending and } \\
\text { sigmoid }\end{array}$ & 6 & Laparoscopic & Discharged \\
\hline 6 & 54 & Female & CT abdomen & $\begin{array}{l}\text { Descending and } \\
\text { sigmoid }\end{array}$ & 4.5 & Endoscopic & Discharged \\
\hline 7 & 86 & Female & Cl abdomen & Sigmoid & 3.7 & Laparotomy & Discharged \\
\hline 8 & 88 & Female & CT abdomen & Sigmoid & 4.4 & Trephine loop colostomy & Discharged \\
\hline
\end{tabular}




\begin{tabular}{|c|c|c|c|c|c|c|c|}
\hline 9 & 78 & Female & CT abdomen & Descending colon & 6 & Laparotomy & Died \\
\hline 10 & 80 & Female & CT abdomen & Sigmoid & 2 & Laparotomy & Discharged \\
\hline 11 & 80 & Female & CT abdomen & Sigmoid & 3 & Laparotomy & Discharged \\
\hline 12 & 92 & Male & CT abdomen & Sigmoid & 3.8 & Laparotomy & Discharged \\
\hline 13 & 94 & Female & CT abdomen & Sigmoid & 6 & Endoscopic with lithotripsy & Discharged \\
\hline 14 & 95 & Female & CT abdomen & Sigmoid & 3.5 & Conservative & Died \\
\hline 15 & 77 & Female & X-Ray abdomen & & 3 & Endoscopy & Unknown \\
\hline 16 & 92 & Male & CT abdomen & Sigmoid & 4.1 & Endoscopy with lithotripsy & Discharged \\
\hline 17 & 85 & Male & CT abdomen & Sigmoid & 4 & Conservative & Discharged \\
\hline 18 & 86 & Male & CT abdomen & Sigmoid & 3.3 & Laparotomy & Discharged \\
\hline 19 & 88 & Female & Laparotomy & Transverse colon & 7 & Laparotomy & Discharged \\
\hline 20 & 49 & Female & CT abdomen & Sigmoid & 4 & Laparotomy & Discharged \\
\hline 21 & 92 & Male & CT abdomen & Descending colon & 2.5 & Laparotomy & Discharged \\
\hline 22 & 73 & Male & CT abdomen & Sigmoid & 4 & Endoscopy with lithotripsy & Discharged \\
\hline 23 & 83 & Female & CT abdomen & Sigmoid & Unknown & Endoscopy laser lithotripsy & Died \\
\hline 24 & 81 & Female & CT abdomen & Sigmoid & 2.5 & Laparotomy & Discharged \\
\hline 25 & 91 & Female & CT abdomen & Sigmoid & 4.6 & Laparotomy & Discharged \\
\hline 26 & 86 & Female & CT abdomen & Sigmoid & 3.7 & Laparotomy & Discharged \\
\hline 27 & 86 & Female & CT abdomen & Sigmoid & 3 & Laparotomy & Discharged \\
\hline 28 & 76 & Female & CT abdomen & Sigmoid & 3.5 & Laparotomy & Discharged \\
\hline 29 & 70 & Female & CT abdomen & Descending colon & 3 & Laparotomy & Died \\
\hline 30 & 85 & Female & Laparotomy & Sigmoid & 6.5 & Laparotomy & Discharged \\
\hline 31 & 80 & Female & Laparotomy & Sigmoid & 2.3 & Laparotomy & Discharged \\
\hline 32 & 78 & Female & X-Ray abdomen & Sigmoid & 4 & Laparotomy & Unknown \\
\hline 33 & 87 & Female & CT abdomen & Sigmoid & 4.2 & Laparotomy & Unknown \\
\hline 34 & 65 & Female & Colonoscopy & Descending colon & Unknown & Conservative & Discharged \\
\hline 35 & 65 & Male & CT abdomen & Sigmoid & 7 & Laparotomy & Discharged \\
\hline 36 & 80 & Female & CT abdomen & Sigmoid & 5 & $\begin{array}{l}\text { Endoscopy with extracorporeal } \\
\text { lithotripsy }\end{array}$ & Discharged \\
\hline 37 & 88 & Female & CT abdomen & Sigmoid & 4.7 & Laparotomy & Died \\
\hline 38 & 68 & Female & CT abdomen & Sigmoid & 6 & Laparoscopic & Discharged \\
\hline 39 & 80 & Female & CT abdomen & Sigmoid & 2 & Laparotomy & Discharged \\
\hline 40 & 72 & Male & CT abdomen & Sigmoid & 4 & Laparotomy & Unknown \\
\hline 41 & 73 & Male & CT abdomen & Transverse Colon & 7 & Laparotomy & Discharged \\
\hline 42 & 89 & Female & CT abdomen & Sigmoid & 5 & Laparotomy & Discharged \\
\hline 43 & 77 & Female & Sigmoidoscopy & Sigmoid & 3.8 & Laparotomy & Unknown \\
\hline 44 & 77 & Female & CT abdomen & Sigmoid & 2.8 & Laparotomy & Discharged \\
\hline 45 & 69 & Female & CT abdomen & Sigmoid & 3.6 & Laparotomy & Discharged \\
\hline 46 & 89 & Female & CT abdomen & Sigmoid & 5 & Laparotomy & Discharged \\
\hline 47 & 69 & Female & CT abdomen & Sigmoid & 4.8 & Manual Removal & Disch \\
\hline
\end{tabular}




\section{Cureus}

$\begin{array}{|llllllll|}48 & 67 & \text { Male } & \text { CT abdomen } & \text { Sigmoid } & 7 & \text { Sigmoidoscopy } & \text { Unknown } \\ 49 & 78 & \text { Male } & \text { CT abdomen } & \text { Sigmoid } & 3 & \text { Laparotomy } & \text { Unknown } \\ 50 & 75 & \text { Female } & \text { CT abdomen } & \text { Sigmoid } & \text { Unknown } & \text { Laparotomy } & \text { Unknown } \\ 51 & 80 & \text { Unknown } & \text { CT abdomen } & \text { Sigmoid } & \text { Unknown } & \text { Surgery } & \text { Unknown } \\ 52 & 87 & \text { Male } & \text { Laparotomy } & \text { Sigmoid } & \text { Unknown } & \text { Laparotomy } & \text { Unknown } \\ 53 & 92 & \text { Female } & \text { CT abdomen } & \text { Transverse colon } & \text { Unknown } & \text { Conservative } & \text { Discharged } \\ 54 & 78 & \text { Male } & \text { X-ray abdomen } & \text { Sigmoid } & \text { Unknown } & \text { Laparotomy } & \text { Discharged } \\ 55 & 87 & \text { Female } & \text { CT abdomen } & \text { Sigmoid } & \text { Unknown } & \text { Laparotomy } & \text { Discharged } \\ 56 & 88 & \text { Female } & \text { Laparotomy } & \text { Sigmoid } & \text { Unknown } & \text { Lapartotomy } & \text { Discharged } \\ 57 & 89 & \text { Male } & \text { CT abdomen } & \text { Sigmoid } & 3.4 & \text { Laparotomy } & \text { Died } \\ \text { (Ours) } & & & & & & & \end{array}$

\section{TABLE 5: Main characteristics of the reported cases of colonic gallstone ileus.}

The main characteristics of the reported cases of colonic gallstone ileus included age, diagnostic modality, stone location and size, treatment, and outcome. When the location of the stone was around the rectosigmoid area it was referred to as sigmoid.

Sigmon et al. used an adult diagnostic gastroscope and performed several attempts to capture the stone with a Roth Net which were unsuccessful, they then used an adult therapeutic gastroscope with a Talon grasping device that was again, unsuccessful. With the last gastroscope and a hexagonal AcuSnare they were able to snare the stone and remove it entirely in one piece [2]. Balzarini et al. used a different approach during their endoscopic procedure, an Olympus videoscope and $\mathrm{CO} 2$ insufflation were utilized, and a 3 x 6 French lithotripsy extraction basket was inserted through the biopsy channel of the videoscope, the stone was caught with the basket, and lithotripsy was successfully performed [11]. Zielinski et al. successfully removed a gallstone from the sigmoid via intracolonic electrohydraulic lithotripsy provided by a French electrohydraulic lithotripsy probe that was also inserted in an Olympus gastroscope [12]. In our case, the several attempts to capture the stone were unsuccessful which could be explained by the degree of stenosis at the site of impaction, furthermore, the mucosa had increased friability and was at risk for perforation.

The overall mortality related to gallstone ileus had been decreasing, In the nineteenth century, gallstone ileus had a high mortality rate of around 44\% [3]. In the large review of 1001 cases by Reisner et al. in 1994, the reported mortality rate had decreased to $18 \%$ [1]. Moreover, the very large study of Halabi et al. showed a mortality rate of $6.67 \%$ [6]. In this review, 48 cases reported the patient's outcome, from these six died during the hospitalization which corresponds to a mortality rate of $12.5 \%$.

\section{Conclusions}

Management of colonic gallstone ileus in high-risk patients is challenging, particularly due to the common advanced age and several comorbidities that many of these patients possess which make them poor surgical candidates. Nonetheless, given the low success rate with other therapeutic modalities, laparotomy remains the standard approach which may involve enterolithotomy or even partial colectomy if the intestinal wall is perforated or necrotic. Endoscopic treatment has been reported in the literature with only a few successful cases mainly using mechanical or electrohydraulic lithotripsy. Several factors influence this outcome including stone size, presence of colonic stricture, time for treatment, and lack of expertise or availability. In our patient, the failure of the endoscopic approach was likely a combination of these factors. An endoscopic approach using the available tools and expertise should be attempted in the presence of surgical support due to the risk of intestinal perforation and often failed endoscopic attempts. Conservative management is not recommended as the spontaneous evacuation of the stone is rare and treatment delays will most likely lead to severe complications.

\section{Additional Information \\ Disclosures}

Human subjects: Consent was obtained or waived by all participants in this study. Conflicts of interest: In compliance with the ICMJE uniform disclosure form, all authors declare the following: Payment/services info: All authors have declared that no financial support was received from any organization for the submitted work. Financial relationships: All authors have declared that they have no financial relationships at present or within the previous three years with any organizations that might have an interest in the submitted work. Other relationships: All authors have declared that there are no other 


\section{Cureus}

relationships or activities that could appear to have influenced the submitted work.

\section{References}

1. Reisner RM, Cohen JR: Gallstone ileus: a review of 1001 reported cases . Am Surg. 1994, 60:441-446.

2. Sigmon L, Rejeski J, Marion B, Fina M: Colonic gallstone ileus. BMJ Case Rep. 2017, 2017:bcr2017220898. 10.1136/bcr-2017-220898

3. Nuño-Guzmán CM, Marín-Contreras ME, Figueroa-Sánchez M, Corona JL: Gallstone ileus, clinical presentation, diagnostic and treatment approach. World J Gastrointest Surg. 2016, 8:65-76. 10.4240/wjgs.v8.i1.65

4. Rodríguez Hermosa JI, Codina Cazador A, Gironès Vilà J, Roig García J, Figa Francesch M, Acero Fernández D: [Gallstone Ileus: results of analysis of a series of 40 patients] . Gastroenterol Hepatol. 2001, 24:489-494.

5. Clavien PA, Richon J, Burgan S, Rohner A: Gallstone ileus. Br J Surg. 1990, 77:737-742. 10.1002/bjs.1800770707

6. Halabi WJ, Kang CY, Ketana N, et al.: Surgery for gallstone ileus: a nationwide comparison of trends and outcomes. Ann Surg. 2014, 259:329- 335. 10.1097/SLA.0b013e31827eefed

7. Athwal TS, Howard N, Belfield J, Gur U: Large bowel obstruction due to impaction of a gallstone . BMJ Case Rep. 2012, 2012:bcr1120115100. 10.1136/bcr.11.2011.5100

8. Anseline P: Colonic gall-stone ileus. Postgrad Med J. 1981, 57:62-65. 10.1136/pgmj.57.663.62

9. Marenco-de la Cuadra B, López-Ruiz JA, Tallón-Aguilar L, López-Pérez J, Oliva-Mompeán F: [Colonic gallstone ileus: a rare cause of intestinal obstruction]. Cir Cir. 2017, 85:440-443. 10.1016/j.circir.2016.05.016

10. Klarenbeek BR, Samuels M, van der Wal MA, van der Peet DL, Meijerink WJ, Cuesta MA: Indications for elective sigmoid resection in diverticular disease. Ann Surg. 2010, 251:670-674. 10.1097/SLA.0b013e3181d3447d

11. Balzarini M, Broglia L, Comi G, Calcara C: Large bowel obstruction due to a big gallstone successfully treated with endoscopic mechanical lithotripsy. Case Rep Gastrointest Med. 2015, 2015:798746. $10.1155 / 2015 / 798746$

12. Zielinski MD, Ferreira LE, Baron TH: Successful endoscopic treatment of colonic gallstone ileus using electrohydraulic lithotripsy. World J Gastroenterol. 2010, 16:1533-1536. 10.3748/wjg.v16.i12.1533 